\title{
Energy-Efficient MISO Systems Using Adaptive Modulation and Coding
}

\author{
Antonio G. Marques*, Xin Wang ${ }^{\dagger}$, and Georgios B. Giannakis ${ }^{\ddagger}$ \\ *Dept. of Signal Theory and Communications. Universidad Rey Juan Carlos. Fuenlabrada, 28943 Madrid (SPAIN) \\ ${ }^{\dagger}$ Dept. of Electrical Engineering. Florida Atlantic University. Boca Raton, 33431 FL (USA) \\ $\ddagger$ Dept. of Electrical and Computer Engineering. University of Minnesota. Minneapolis, 55455 MN (USA) \\ Emails: antonio.garcia.marques@urjc.es, xin.wang@fau.edu, georgios@ece.umn.edu
}

\begin{abstract}
By viewing the coherent wireless sensor network (WSN) setup as a distributed space-time multi-input singleoutput (MISO) system, we minimize average transmit-power when sensors communicate with a fusion center (FC) using adaptive modulation and coding over a wireless fading channel. To this end, we derive optimal distributed beamforming and resource allocation strategies when the full (F-) channel state information at the transmitters (CSIT) is available, or, each sensor has F-CSIT of its own link with the FC but only quantized CSIT of other sensors through finite-rate feedback. Numerical results are presented to evaluate the power savings of the novel strategies.
\end{abstract}

\section{INTRODUCTION}

Wireless sensor network (WSN) is an attractive low-cost technology for a wide range of remote sensing and environmental monitoring applications [1]. A main objective of current WSN research is to design power-efficient devices and algorithms to support different aspects of network operations [4]. Various power-efficient algorithms have been proposed for network coverage, medium access control protocols, decentralized estimation and routing; see e.g., [4], [7], [10], [9], and [2]. The WSN in many of these works includes a fusion center (FC) with which sensors are linked.

By viewing the coherent wireless sensor network (WSN) setup as a distributed space-time multi-input single-output (MISO) system [8], in this paper we consider minimizing the average transmit-power in a WSN, where sensors communicate with a FC using adaptive modulation-coding (AMC) over a wireless fading channel. Specifically, the sensors' average transmit-power is minimized subject to average rate and bit error rate (BER) constraints, based on two different types of channel state information at transmitters (CSIT): i) Full (F-) CSIT where each channel realization is assumed available at each sensor; and ii) Individual (I-) CSIT where each sensor has full knowledge of its own channel but only quantized knowledge of the other sensors' channels through finite-rate feedback. For these cases, we derive the corresponding optimal adaptive modulation/coding, power loading and beamforming strategies as well as optimal channel quantizers needed to form the required quantized (Q-) CSIT feedback.

Notation: We use boldface lower-case letters to denote column vectors, ${ }^{T}$ to denote transposition, ${ }^{\dagger}$ conjugate, $\mathcal{H}$ conjugate transposition, and $\|\cdot\|$ the Euclidean norm. For a random variable $x, f_{x}(x)$ will denote its probability density function (PDF), $F_{x}(x)$ its cumulative distribution function $(\mathrm{CDF}), \mathcal{C N}\left(\mu, \sigma^{2}\right)$ the complex-Gaussian distribution with mean $\mu$ and variance $\sigma^{2},\lceil x\rceil$ the minimum integer $\geq x$, and $E_{x}[\cdot]$ the expectation operator over $x$.

\section{Modeling Preliminaries}

We consider a WSN where $M$ sensors indexed by $m \in$ $\{1, \ldots, M\}$ wish to communicate an information message to the FC, under following operating conditions: (oc1) the information is common to all sensors and arrives coherently at the $F C$. With $\left\{h_{m}\right\}_{m=1}^{M}$ denoting block fading channel coefficients between sensors and the FC, we further assume that: (oc-2) $\left\{h_{m}\right\}_{m=1}^{M}$ are independent and identically distributed (i.i.d.) according to a complex Gaussian distribution with zero mean and unit variance, i.e., $h_{m} \sim \mathcal{C N}(0,1)$.

We suppose that each sensor supports a finite number $L$ of AMC modes indexed by $l \in\{1, \ldots, L\}$, with each mode having constellation size $M_{l}$ and transmission rate $r_{l}:=$ $\log _{2}\left(M_{l}\right)$, which must be delivered with a prescribed BER $\epsilon_{0}$. To mitigate the effects of fading, the sensors beamform their transmitted symbol. Since a MISO system has multiplexing gain one (see e.g., [5, pp. 48]), the sensors encode one information-bearing symbol $s$ per channel use utilizing a common AMC mode. With this AMC mode, the $m$ th sensor transmits $s$ multiplied by a complex (steering) weight $w_{m}$. Let $\mathbf{w}:=\left[w_{1}, \ldots, w_{M}\right]^{T}$ denote the distributed beamforming vector and $\mathbf{h}:=\left[h_{1}, \ldots, h_{M}\right]^{T}$ the fading MISO channel. The received symbol $y$ at the $\mathrm{FC}$ can be expressed as

$$
y=\mathbf{w}^{T} \mathbf{h} s+v:=\|\mathbf{w}\|^{2} \mathbf{u}^{T} \mathbf{h} s+v
$$

where $\mathbf{u}:=\mathbf{w} /\|\mathbf{w}\|$, and $v$ denotes the additive white Gaussian noise (AWGN) with zero mean and variance $N_{0}$. Notice that both the phase and the modulus of $\mathbf{w}$ can be tuned to effect not only distributed beamforming but also power allocation per fading state $\mathbf{h}$. Letting $E_{s}$ denote the average energy per symbol, we can write the total transmit-power and receive-SNR per symbol as

$$
p:=\|\mathbf{w}\|^{2} E_{s}=\|\mathbf{w}\|^{2},
$$




$$
\gamma:=\left|\mathbf{w}^{T} \mathbf{h}\right|^{2} \frac{E_{s}}{N_{0}}=p\left|\mathbf{u}^{T} \mathbf{h}\right|^{2},
$$

where for the last equality in (2) and (3) we have assumed without loss of generality that $E_{s}=N_{0}=1$. It follows from (3) that after beamforming, the MISO vector channel $\mathbf{h}$ in (1) is fully characterized by the equivalent SISO scalar channel with normalized power gain $g:=\left|\mathbf{u}^{T} \mathbf{h}\right|^{2}$. The receive-SNR for the equivalent SISO system can be re-writen as $\gamma=p g$.

Our goal is to optimally design the channel quantizer which yields $\mathbf{c}(\mathbf{h})$ based on which we wish to adapt $r=r(\mathbf{c}), p=$ $p(\mathbf{c})$, and $\mathbf{u}=\mathbf{u}(\mathbf{c})$, so that the total average power transmitted by all sensors is minimized subject to average rate and BER requirements.

\section{Solution BASEd ON F-CSIT}

In this section, we derive the optimal adaptive transmission policy based on F-CSIT. Given F-CSIT $\mathbf{h}$, we wish to adapt the transmit-power $p(\mathbf{h})$, rate $r(\mathbf{h})$ and beamforming vector $\mathbf{u}(\mathbf{h})$ to minimize the average transmit-power subject to prescribed requirements on the average rate $\left(r_{0}\right)$ and $\operatorname{BER}\left(\epsilon_{0}\right)$. As we show next, the adaptation of the beamformer can be performed separately from the power and rate adaptation without loss of optimality (w.l.o.o.). This allows us to tackle the original problem in two separate phases: first we solve for the optimal ${ }^{1}$ adaptation of the beamformer $\mathbf{u}^{*}(\mathbf{h})$; next we introduce $\mathbf{u}^{*}(\mathbf{h})$ in the original problem and solve for the optimal power $p^{*}(\mathbf{h})$ and rate $r^{*}(\mathbf{h})$ adaptation.

\section{A. Optimal Distributed Beamformer}

From (2) and (3) we recognize that the selection of $\mathbf{u}$ affects the scalar channel gain $g$. Since for any AMC mode the required transmit power $p=p(\mathbf{h})$ is monotonically decreasing w.r.t. $g$ (for any given $r_{0}$ and $\epsilon_{0}$ ), to minimize the transmitpower we have to adapt $\mathbf{u}=\mathbf{u}(\mathbf{h})$ per channel realization $\mathbf{h}$ so that $g=\left|\mathbf{u}^{T}(\mathbf{h}) \mathbf{h}\right|^{2}$ is maximized. The optimal unitary beamforming vector maximizing $\left|\mathbf{u}^{T}(\mathbf{h}) \mathbf{h}\right|^{2}$, hence minimizing the required transmit-power, is clearly

$$
\mathbf{u}^{*}(\mathbf{h})=\mathbf{h}^{\dagger} /\|\mathbf{h}\|
$$

To proceed with the optimal rate and power allocation strategies, we need to characterize statistically the channel in (1) when beamforming $\mathbf{u}^{*}(\mathbf{h})$ is adapted. When $\mathbf{u}^{*}(\mathbf{h})=$ $\mathbf{h}^{\dagger} /\|\mathbf{h}\|, \forall \mathbf{h}$, the channel gain is

$$
g=\left|\mathbf{u}^{T}(\mathbf{h}) \mathbf{h}\right|^{2}=\|\mathbf{h}\|^{2} .
$$

As per (as2), $g$ adheres to a chi-squared distribution with PDF $f_{g}(g)=\frac{g^{M-1} \exp (-g)}{\Gamma(M)}$, where $\Gamma(b, x):=\int_{x}^{\infty} t^{b-1} e^{-t} d t$ is the incomplete Gamma function and $\Gamma(b):=\Gamma(b, 0)$. The corresponding $\mathrm{CDF}$ is $F_{g}(g)=\frac{\Gamma(M, g)}{\Gamma(M)}$.

It is worth to recall that when optimal beamforming $\mathbf{u}^{*}(\mathbf{h})$ is implemented, the MISO channel in (1) is fully characterized by an equivalent SISO channel with power gain $g$ dictated by (5). This implies w.l.o.o. that solving for the optimal $r^{*}(\mathbf{h})$ and $p^{*}(\mathbf{h})$ is equivalent to finding the optimal $r^{*}(g)$ and $p^{*}(g)$.

\footnotetext{
${ }^{1}$ Henceforth, $x^{*}$ will denote the optimal value of $x$
}

Notice that since $\mathbf{h}$ (and thus $g$ ) varies from one realization to the next, rate and power will be adapted across time in order to minimize the average transmit-power under an average rate constraint $r_{0}$.

\section{B. Optimal Rate and Power Allocation}

We order the AMC modes such that $r_{l}<r_{l+1} \forall l>1$ and let the first mode represent the inactive mode with zero rate and power $\left(r_{1}=p_{1}=0\right)$. With $\epsilon(\cdot)$ denoting the instantaneous BER function, the minimum transmit-power for the $l$ th AMC mode to satisfy the BER requirement $\epsilon_{0}$ can be calculated by solving with respect to (w.r.t.) $p_{l}$ the equation

$$
\epsilon\left(g, p_{l}, r_{l}\right)=\epsilon_{0}
$$

For $M$-ary quadrature amplitude modulation (QAM), the BER can be accurately approximated as [6] (e.g., $\kappa_{1}=0.2, \kappa_{2}=$ 1.5 for uncoded transmissions)

$$
\epsilon(g, p, r)=\kappa_{1} \exp \left(-\kappa_{2} g p /\left(2^{r}-1\right)\right) .
$$

Substituting (7) into (6), the required power for the $l$ th AMC mode can be expressed as

$$
p_{l}\left(g, r_{l}, \epsilon_{0}\right)=\frac{\left(2^{r_{l}}-1\right)}{g} \frac{\ln \left(\kappa_{1} / \epsilon_{0}\right)}{\kappa_{2}} .
$$

With F-CSIT available, (8) shows that specifying the AMC mode determines not only the rate but also the power required to meet the prescribed $\epsilon_{0}$. Furthermore, it is easy to see that with $p_{l}, r_{l}$ given the range of $g$ can be divided into $L$ consecutive intervals $\left[\tau_{l}, \tau_{l+1}\right)$ with $\tau_{1}=0$ and $\tau_{L+1}=\infty$, and the $l$ th $A M C$ mode will be chosen if $g \in\left[\tau_{l}, \tau_{l+1}\right)$. This means that once the intervals $\left[\tau_{l}, \tau_{l+1}\right)$ are specified for $l=1, \ldots, L$, the rate and power allocations will be

$$
\begin{aligned}
r(g) & =r_{l} ; \quad \text { if } g \in\left[\tau_{l}, \tau_{l+1}\right) \\
p\left(g, \epsilon_{0}\right) & = \begin{cases}0, & g \in\left[\tau_{1}, \tau_{2}\right) \\
\frac{\left(2^{r_{l}-1}\right)}{g} \frac{\ln \left(\kappa_{1} / \epsilon_{0}\right)}{\kappa_{2}}, & g \in\left[\tau_{l}, \tau_{l+1}\right), l>1 .\end{cases}
\end{aligned}
$$

Letting $\tau:=\left[\tau_{1}, \ldots, \tau_{L+1}\right]^{T}$, (9) and (10) imply that to find the optimal rate and power allocations, we only need to search for the optimal $\tau^{*}$ which solves the following constrained minimization problem

$$
\left\{\begin{array}{c}
\min _{\tau} \bar{p}, \text { where } \bar{p}:=\sum_{l=1}^{L} \int_{\tau_{l}}^{\tau_{l+1}} p_{l}\left(g, r_{l}, \epsilon_{0}\right) f_{g}(g) d g \\
\text { subject to : } C 1 . \sum_{l=1}^{L} \int_{\tau_{l}}^{\tau_{l+1}} r_{l} f_{g}(g) d g \geq r_{0} \\
C 2 . \tau_{l} \leq \tau_{l+1} \forall l
\end{array}\right.
$$

Using the necessary Karush-Kuhn-Tucker (KKT) condition, we can show that the optimal $\tau_{l}^{*}$ is given by

$$
\tau_{l}^{*}=\frac{\left(2^{r_{l}}-2^{r_{l-1}}\right)}{\lambda^{*}\left(r_{l}-r_{l-1}\right)} \frac{\ln \left(\kappa_{1} / \epsilon_{0}\right)}{\kappa_{2}} ;
$$

where $\lambda^{*}$ denote the non-negative optimal Lagrange multiplier associated with the rate constraint $C 1$. With $\tau_{l}^{*}$ specified by (12), $\lambda^{*}$ can be calculated to satisfy $C 1$ using the following 
algorithm.

Algorithm 1: Off-line Power-Efficient Quantization (F-CSIT)

(S1.0) Let $\delta$ be a small tolerance level and initialize $\lambda$ with an arbitrary positive number.

(S1.1) Calculate $\left\{\tau_{l}\right\}_{l=2}^{L}$ via (12).

(S1.2) Calculate the average rate as $\bar{r}=\sum_{l=1}^{L}\left[F_{g}\left(\tau_{l+1}\right)-\right.$ $\left.F_{g}\left(\tau_{l}\right)\right] r_{l}$, and check $C 1$. If $\left|\bar{r}-r_{0}\right| / r_{0}<\delta$ then stop; otherwise, calculate $\triangle \lambda:=\left(\bar{r}-r_{0}\right) c$, update the multiplier as $\lambda=\lambda+\triangle \lambda$, and go to (S1.1). Parameter $c$ in the calculation of $\triangle \lambda$ is an adaptive penalty parameter that can be updated (per iteration) depending on convergence requirements [3].

Once $\lambda^{*}$ is obtained off-line using Algorithm $1,\left\{\tau_{l}^{*}\right\}_{l=2}^{L}$ and in turn the optimal rate and power allocations are determined after plugging (12) into (9) and (10).

\section{On-line Feedback and Adaptation of Transmitters}

Having obtained $\left\{\tau_{l}^{*}\right\}_{l=2}^{L}$, the following algorithm summarizes the on-line resource allocation steps the WSN has to execute per channel realization:

\section{Algorithm 2: On-line Adaptation (F-CSIT)}

For each channel realization $\mathbf{h}$ :

(S2.1) The FC determines the index $l^{*}(\mathbf{h})=l^{*}(g)$ of the interval $\left[\tau_{l}^{*}, \tau_{l+1}^{*}\right)$ the channel gain $g$ falls into, and broadcasts to the sensors the F-CSIT codeword $\mathbf{c}((\mathbf{h}))=\left[l^{*}(\mathbf{h}) ; \mathbf{h}\right]$.

(S2.2) Each sensor $m$ transmits using the $l^{*}(\mathbf{h})$ th AMC mode and the optimal steering weight [cf. (4) and (8)] $w_{m}^{*}=$ $\sqrt{p_{l^{*}(\mathbf{h})}\left(g, r_{l^{*}(\mathbf{h})}, \epsilon_{0}\right)} h_{m}^{\dagger} /\|\mathbf{h}\|$.

Notice that even though each sensor can calculate $l^{*}(\mathbf{h})$ using only $\mathbf{h}$ of the feedback message, we also include $l^{*}(\mathbf{h})$ in $\mathbf{c}(\mathbf{h})$ for robustness. This augmented feedback codeword reduces the computational burden at each sensor. With the insights gained from the F-CSIT based benchmark, we next derive the optimal adaptation schemes when only I-CSIT is available.

\section{SOlution BASEd ON I-CSIT}

F-CSIT of the whole vector channel $\mathbf{h}$ at each sensor cannot be pragmatically affordable in practical systems. In time-division duplex (TDD) systems, each sensor $m$ can only acquire accurate F-CSIT $h_{m}$ of its own channel via pilot-based channel estimation during the symmetric reverse transmission. However, sensors can only obtain the CSI of others' channel through a limited-rate feedback from the FC. This motivates analysis of what we call individual (I-) CSIT scenario, where each sensor $m$ has full knowledge of $h_{m}$, but only quantized version of the other entries in $\mathbf{h}$.

\section{A. Optimal Distributed Beamforming}

Defining $\mathbf{v}:=\|\mathbf{h}\| \mathbf{u}$ and $\rho:=p /\|\mathbf{h}\|^{2}$, we can write $\mathbf{w}=\sqrt{p} \mathbf{u}=\sqrt{\rho} \mathbf{v}$. When F-CSIT is available, the optimal beamforming $\mathbf{u}^{*}(\mathbf{h})$ is given by (4) and thus the $m$ th entry of the optimal $\mathbf{v}^{*}(\mathbf{h})=\mathbf{h}^{\dagger}$ is $v_{m}^{*}(\mathbf{h})=h_{m}^{\dagger}$, which requires only I-CSIT. The receive-SNR after optimal beamforming based on I-CSIT is $\gamma=\rho g^{2}=p g$, where $g$ corresponds to the equivalent chi-squared distributed SISO channel gain in (5).

\section{B. Optimal Rate and Power Quantization and Allocation}

Given I-CSIT, the optimal (scaled) beamformer is $\mathbf{v}^{*}(\mathbf{h})$. To construct the entire steering vector $\mathbf{w}(\mathbf{h})$, the sensors need also the (scaled) transmit-power $\rho(\mathbf{h})=p(\mathbf{h}) /\|\mathbf{h}\|^{2}$ which requires knowledge of the whole channel $\mathbf{h}$ (or equivalently $\rho(g):=p(g) / g$ ). With finite-rate feedback, the FC quantizes the channel gain $g$ using a finite number of regions; i.e., $L$ different quantization regions $\left\{\mathcal{R}_{l}:=\left[\tilde{\tau}_{l}, \tilde{\tau}_{l+1}\right)\right\}_{l=1}^{L}$ so that the $l$ th AMC mode is employed by the sensors when $g \in \mathcal{R}_{l}$. Therefore, the $l$ th AMC mode is characterized by the ratepower pair $\left(r_{l}, \tilde{\rho}_{l}\right)$, where $\tilde{\rho}_{l}$ is fixed per region and must be selected to satisfy the BER requirement $\epsilon_{0} \cdot{ }^{2}$ Upon defining

$$
\psi_{\epsilon}\left(\tilde{\tau}_{l}, \tilde{\tau}_{l+1}, \tilde{\rho}_{l}, r_{l}, \epsilon_{0}\right):=\int_{\tilde{\tau}_{l}}^{\tilde{\tau}_{l+1}}\left(\epsilon\left(g, \tilde{\rho}_{l} g, r_{l}\right)-\epsilon_{0}\right) f_{g}(g) d g,
$$

we can show that a $\tilde{\rho}_{l}$ satisfying the prescribed BER (denoted by $\left.\tilde{\rho}_{l}\left(\tilde{\tau}_{l}, \tilde{\tau}_{l+1}, r_{l}, \epsilon_{0}\right)\right)$ should solve $\psi_{\epsilon}\left(\tilde{\tau}_{l}, \tilde{\tau}_{l+1}, \tilde{\rho}_{l}, r_{l}, \epsilon_{0}\right)=0$. Relying on the fact that $\epsilon\left(g, \tilde{\rho}_{l} g, r_{l}\right)$ is monotonically decreasing w.r.t. to $\tilde{\rho}_{l}$, the root $\tilde{\rho}_{l}\left(\tilde{\tau}_{l}, \tilde{\tau}_{l+1}, r_{l}, \epsilon_{0}\right)$ can be efficiently obtained via one-dimensional search.

We can now proceed to optimize resource allocation based on I-CSIT. Given $\tilde{\rho}_{l}\left(\tilde{\tau}_{l}, \tilde{\tau}_{l+1}, r_{l}, \epsilon_{0}\right)$ and a realization $\mathbf{h}$, the transmit-power when the $l$ th AMC mode is selected can be found as $p(\mathbf{h})=\|\mathbf{h}\|^{2} \tilde{\rho}_{l}\left(\tilde{\tau}_{l}, \tilde{\tau}_{l+1}, r_{l}, \epsilon_{0}\right)=$ $g \tilde{\rho}_{l}\left(\tilde{\tau}_{l}, \tilde{\tau}_{l+1}, r_{l}, \epsilon_{0}\right)=p(g)$. To find the optimal quantization thresholds $\tilde{\tau}^{*}:=\left[\tilde{\tau}_{1}^{*}, \ldots, \tilde{\tau}_{L+1}^{*}\right]^{T}$ minimizing the average transmit-power, we need to solve

$$
\left\{\begin{array}{c}
\min _{\tilde{\tau}} \bar{p}, \text { where } \bar{p}:=\sum_{l=1}^{L} \tilde{\rho}_{l}\left(\tilde{\tau}_{l}, \tilde{\tau}_{l+1}, r_{l}, \epsilon_{0}\right) \int_{\tilde{\tau}_{l}}^{\tilde{\tau}_{l+1}} g f_{g}(g) d g \\
\text { subject to : } C 1 \cdot \sum_{l=1}^{L} r_{l} \int_{\tilde{\tau}_{l}}^{\tilde{\tau}_{l+1}} f_{g}(g) d g \geq r_{0} \\
C 2 . \tilde{\tau}_{l} \leq \tilde{\tau}_{l+1} \forall l .
\end{array}\right.
$$

Letting $\tilde{\lambda}$ denote the Lagrange multiplier associated with $C 1$ and assuming all the constraints in $C 2$ are satisfied with strict inequality, the KKT condition for the optimal $\tilde{\tau}_{l}^{*}$ yields (15), where $\int_{a}^{b} g f_{g}(g) d g=[\Gamma(M+1, a)-\Gamma(M+1, b)] / \Gamma(M)$; and $\partial \tilde{\rho}_{i} / \partial \tilde{\tau}_{l}, \forall l \in[2, L], \forall i \in[1, L]$, can be obtained through

\footnotetext{
${ }^{2}$ Note that here $\tilde{\rho}_{l}$ is fixed and constant per region (i.e., $\tilde{\rho}$ only changes as the region index $l$ (Q-CSIT) changes), as apposed to what happens in Section III where $p_{l}$ is adapted as a function of $\|\mathbf{h}\|$ (i.e., $p$ changes as $\|\mathbf{h}\|$ (F-CSIT) changes).
} 


$$
\begin{array}{r}
\frac{\partial \mathcal{L}\left(\tilde{\lambda}^{*}, \tilde{\boldsymbol{\tau}}^{*}\right)}{\partial \tau_{l}}=\left[\tilde{\tau}_{l}^{*} \tilde{\rho}_{l-1}\left(\tilde{\tau}_{l-1}^{*}, \tilde{\tau}_{l}^{*}, r_{l-1}, \epsilon_{0}\right)-\tilde{\lambda}^{*} r_{l-1}-\tilde{\tau}_{l}^{*} \tilde{\rho}_{l}\left(\tilde{\tau}_{l}^{*}, \tilde{\tau}_{l+1}^{*}, r_{l}, \epsilon_{0}\right)+\tilde{\lambda}^{*} r_{l}\right] f_{g}\left(\tilde{\tau}_{l}^{*}\right) \\
+\frac{\partial \tilde{\rho}_{l-1}}{\partial \tilde{\tau}_{l}}\left(\tilde{\tau}_{i}, \tilde{\tau}_{i+1}, r_{i}, \epsilon_{0}\right) \int_{\tilde{\tau}_{l-1}^{*}}^{\tilde{\tau}_{l}^{*}} g f_{g}(g) d g+\frac{\partial \tilde{\rho}_{l}}{\partial \tilde{\tau}_{l}}\left(\tilde{\tau}_{i}, \tilde{\tau}_{i+1}, r_{i}, \epsilon_{0}\right) \int_{\tilde{\tau}_{l}^{*}}^{\tilde{\tau}_{l+1}^{*}} g f_{g}(g) d g=0
\end{array}
$$

implicit differentiation as

$$
\begin{aligned}
& \frac{\partial \tilde{\rho}_{i}}{\partial \tilde{\tau}_{l}}\left(\tilde{\tau}_{i}, \tilde{\tau}_{i+1}, r_{i}, \epsilon_{0}\right)= \\
& \begin{cases}-\frac{\left[-\epsilon\left(\tilde{\tau}_{l}, \tilde{\tau}_{l} \tilde{\rho}_{i}, r_{l}\right)+\epsilon_{0}\right] f_{g}\left(\tilde{\tau}_{l}\right)}{\int_{\tilde{\tau}_{i}+1}^{\tilde{\tau}_{i}}\left[\partial \epsilon\left(g, g \tilde{\rho}_{i}, r_{l}\right) / \partial p\right] g f_{g}(g) d g}, & i=l \\
\frac{\left[-\epsilon\left(\tilde{\tau}_{l}, \tilde{\tau}_{l} \tilde{\rho}_{i}, r_{l-1}\right)+\epsilon_{0}\right] f_{g}\left(\tilde{l}_{l}\right)}{\tilde{\tau}_{\tilde{\tau}_{i}+1}^{\tilde{\tau}_{i+1}}\left[\partial \epsilon\left(g, g \tilde{\rho}_{i}, r_{l-1}\right) / \partial p\right] g f_{g}(g) d g}, & i=l-1 \\
0, & \text { otherwise. }\end{cases}
\end{aligned}
$$

Notice that calculating the optimal $\tilde{\tau}_{l}^{*}$ here depends not only on $\tilde{\lambda}^{*}$ but also on the previous $\tilde{\tau}_{l-1}^{*}$ and the next $\tilde{\tau}_{l+1}^{*}$. This prevents one from obtaining a closed-form expression for $\tilde{\tau}_{l}^{*}$. However, since closed-form expressions for all the terms in (15) are available, $\tilde{\tau}_{l}^{*}$ can be obtained numerically using a two-dimensional search which is computationally affordable.

Algorithm 3: Off-line Power-Efficient Quantization (I-CSIT)

(S3.0) Let $\delta$ denote a small tolerance, $\varepsilon$ a small step size, and $\tilde{\tau}_{L}^{\max }>0$ the maximum value for the highest quantization threshold (e.g., a value bringing the probability of the highest region close to 0 ).

(S3.1) Initialize $\tilde{\lambda}$ with a small positive number and set $\tilde{\tau}_{L}=$ $\tilde{\tau}_{L}^{\max }$; then calculate $\left\{\tilde{\tau}_{l}\right\}_{l=2}^{L}$ by solving (15). If $C 2$ is not satisfied for some $\tau_{l}$, set $\tau_{l}=\tau_{l+1}$. If the obtained solution is feasible, go to (S3.2); otherwise decrease $\tilde{\tau}_{L}=$ $\tilde{\tau}_{L}-\varepsilon$ and repeat $(\mathrm{S} 3.1)$.

(S3.2) Calculate the average rate $\bar{r}=\sum_{l=1}^{L}\left[F_{g}\left(\tilde{\tau}_{l+1}\right)-\right.$ $\left.F_{g}\left(\tilde{\tau}_{l}\right)\right] r_{l}$. Check $C 1$ and if $\left|\bar{r}-r_{0}\right| / r_{0}<\delta$ then stop; otherwise, calculate $\Delta \tilde{\lambda}:=\left(\bar{r}-r_{0}\right) c$ using a small positive adaptive constant $c$, update the multiplier to $\tilde{\lambda}=\tilde{\lambda}+\triangle \tilde{\lambda}$, and go back to (S3.1).

\section{On-line Feedback and Adaptation of Transmitters}

Once the optimal thresholds $\tilde{\tau}^{*}$ are obtained, the corresponding $\tilde{\rho}_{l}\left(\tilde{\tau}_{l}^{*}, \tilde{\tau}_{l+1}^{*}, r_{l}, \epsilon_{0}\right)$ can be computed at both the FC and the sensors, which then implement the following on-line algorithm to adapt their transmissions per channel realization:

\section{Algorithm 4: On-line Channel Adaptation (I-CSIT)}

For each channel realization $\mathbf{h}$ :

(S5.1) The FC finds $l^{*}(\mathbf{h})=l^{*}(g)=\underset{l}{\arg }\left\{g \in\left[\tilde{\tau}_{l}^{*}, \tilde{\tau}_{l+1}^{*}\right)\right\}$, and broadcasts $\mathbf{c}=\left[l^{*}(\mathbf{h})\right]$ to all sensors.
(S5.2) Each sensor $m$ transmits the common symbol $s$ using the $l^{*}(\mathbf{h})$ th $\mathrm{AMC}$ mode and steering weigh $w_{m}^{*}=$ $\sqrt{\rho_{l^{*}(\mathbf{h})}\left(\tilde{\tau}_{l^{*}(\mathbf{h})}^{*}, \tilde{\tau}_{l^{*}(\mathbf{h})+1}^{*}, r_{l^{*}(\mathbf{h})}, \epsilon_{0}\right)} h_{m}^{\dagger}$.

Notice that since the beamformer based on I-CSIT does not require feedback from the $\mathrm{FC}$, we only need $B=\log _{2}(L)$ bits for CSI feedback.

\section{Simulations}

In this section, we present numerical examples to assess the transmit-power consumed by the sensors when F-CSIT, or ICSIT is available. The energy per symbol, system bandwidth and AWGN power spectral density are selected to satisfy $E_{s} / N_{0}=1$. The simple cases tested include four sensors with fading links adhering to (oc-1). Unless otherwise specified, we suppose that each sensor supports three active $M$-ary QAM uncoded modes: 2-QAM, 8-QAM and 32-QAM plus the inactive state; i.e., the transmission rates of AMC modes are: $r_{l}=0,1,3,5$ bits/symbol. In all simulations, we set the BER requirement to $\epsilon_{0}=10^{-3}$.

For variable rate requirements, Fig. 1 shows the average transmit-power (measured in $W$-watts for the upper plot-, and in $d B_{W}$-decibels w.r.t. 1 watt for the lower plot-) achieved by the optimal adaptation policies based on: (i) F-CSIT, (ii) I-CSIT, (iii) spatial (S-) CSIT, and no CSIT. For comparison and illustration purposes in the third S-CSIT case we consider that the sensors implement optimal spatial beamforming based on F-CSIT but do not implement temporal power allocation across time, while in the fourth case we consider a nonadaptive equal power and rate allocation. From Fig. 1, we have the following interesting observations: (i) I-CSIT based strategies can achieve power efficiency close to the optimal FCSIT based one; (ii) I-CSIT based strategy clearly outperforms the optimal S-CSIT scheme although the latter requires infinite feedback rate while the former only require of few bits of feedback; and (iii) the savings relative to the case when no adaptation is implemented are as high as $20 \mathrm{~dB}$.

We have seen that with $L=4$ (three active AMC modes), the I-CSIT based solutions yield power efficiency close to the optimal F-CSIT benchmark. This is achieved using $\left\lceil\log _{2}(4)\right\rceil=2$ bits per channel realization. Next, we analyze how the number of feedback bits affects the performance by varying the number of supported AMC modes. Table I lists the total power cost in the F-CSIT and I-CSIT cases for different $L$ values. (When $L=1$, the sensors only support one AMC mode which does not require feedback; whereas for all the remaining cases, sensors support $L-1$ active AMC modes 


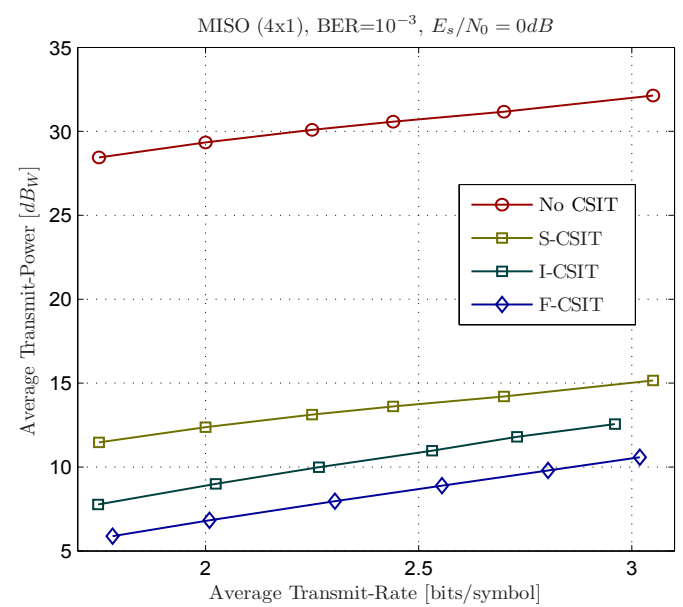

Fig. 1. Total transmit-power $(\bar{p})$ vs total transmit-rate for different CSIT scenarios $(M=4, L=4)$.

TABLE I

AVERAGE TRANSMIT-POWER (IN $d B_{W}$ ) AS $L$ VARIES.

\begin{tabular}{|c|c|c|c|c|c|c|}
\hline \hline$L$ & 1 & 2 & 4 & 6 & 8 & $\infty$ \\
\hline \hline F-CSIT & 9.8 & 9.4 & 8.6 & 8.5 & 8.4 & 8.3 \\
\hline I-CSIT & 13.9 & 11.5 & 10.2 & 9.4 & 8.9 & 8.3 \\
\hline \hline
\end{tabular}

plus an inactive mode indexed by feeding back $\left\lceil\log _{2}(L)\right\rceil$ bits.) As $L$ increases, we observe that: (i) the power consumption decreases for all solutions, (ii) the power-gap of the I-CSIT based systems from the F-CSIT benchmark also decreases, and (iii) the first and second increments of $L$ bring the largest power savings. Clearly, although theoretically the power gap of I-CSIT relative to F-CSIT tends to zero as $L, N_{u} \rightarrow \infty$, our numerical results suggest that even a few feedback bits (e.g., $L=2^{3}$ ) suffice to close the gap.

\section{CONCLUSIONS}

In a WSN entailing coherent sensor communications with a fusion center, we minimized the average transmit-power subject to average rate and BER requirements when full (F-) CSIT or individual (I-) CSIT is available. With finiterate feedback, we optimally separated the main design in two subproblems: (i) MISO channel quantization and beamforming, and (ii) rate/power quantization and allocation. By exploiting the parallelism between the coherent WSN setup and a distributed MISO system, we relied on non-linear programming tools to solve the programs at hand and derived the corresponding power-efficient channel quantization and adaptive transmission policies. Numerical results confirmed that our limited-rate feedback I-CSIT based solution attains power efficiency surprisingly close to the optimal F-CSIT based benchmark, and outperforms a S-CSIT scheme which only exploits spatial diversity with F-CSIT. ${ }^{3}$

\section{REFERENCES}

[1] I. F. Akyildiz, W. Su, Y. Sankarsubramaniam, and E. Cayirci, "Wireless sensor networks: A survey," Computer Networks, vol. 38, pp. 393-422, Mar. 2002.

[2] J. N. Al-Karaki and A. E. Kamal, "Routing techniques in wireless sensor networks: A survey," IEEE Wireless Commun. Mag., vol. 11, no. 6, pp. 6-28, Dec. 2004.

[3] D. Bertsekas, Nonlinear Programming: 2nd Ed., Athena Scientific, 1999.

[4] M. Bhardwaj, T. Garnett, and A. P. Chandrakasan, "Upper bounds on the lifetime of sensor networks," Proc. of IEEE Int. Conf. Commun., vol. 3, Helsinki, Finland, Jun. 2001, pp. 785-790.

[5] G. B. Giannakis, Z. Liu, X. Ma, and S. Zhou, Space-Time Coding for Broadband Wireless Communications, John Wiley and Sons, Inc., 2006.

[6] A. J. Goldsmith and S. G. Chua, "Adaptive coded modulation for fading channels," IEEE Trans. on Commun., vol. 46, pp. 595-602, May 1998.

[7] A. G. Marques, X. Wang and G. B. Giannakis, "Energy-efficient TDMA with quantized channel state information," Proc. of MILCOM Conf., Washington, DC, Oct. 2006.

[8] W. U. Bajwa, A. M. Sayeed, and R. Nowak, "Matched source-channel communication for field estimation in wireless sensor networks," Proc. 4th Intl. Conf. on Inform. Processing in Sensor Networks, Los Angeles, CA, Apr. 2005, pp. 332-339.

[9] J.-J. Xiao, S. Cui; Z.-Q. Luo; A.J. Goldsmith, "Power scheduling of universal decentralized estimation in sensor networks," IEEE Trans. on Sig. Process., vol. 54, no. 2, pp. 413-422, Feb. 2006.

[10] W. Ye, J. Heidemann, and D. Estrin, "An energy-efficient MAC protocol for wireless sensor networks," Proc. of 21st Annual Joint Conf. IEEE Computer Commun. Societies, vol. 3, New York, Jun. 2002, pp. 15671576 .

\footnotetext{
${ }^{3}$ The views and conclusions contained in this document are those of the authors and should not be interpreted as representing the official policies, either expressed or implied, of the Army Research Laboratory or the U. S. Government.
} 